\title{
Mastering the design for rehabilitation strategies in ICU survivors
}

\author{
Peter E Morris, Ashley Montgomery-Yates
}

Although the data from several recent trials have informed our understanding of early intensive care unit (ICU) exercise treatment, there a distinct lack of clarity is terms of the dose, duration and frequency of exercise therapy that should be delivered to the ICU survivor. ${ }^{1-4}$ Indeed, the coordination and integration of care for the critically ill patient, from early ICU exercise therapy and mobilisation to post-ICU rehabilitation, will be key going forward. In this month's Thorax, McDowell et $a l^{5}$ report on the REVIVE trial, which provides insight into the management of the post-ICU survivor and, in particular, the effectiveness of exercise on physical function (PF) in patients discharged from hospital following critical illness. The target population had received invasive ventilation for at least 4 days on the ICU with the exercise intervention delivered in the outpatient setting after hospital discharge. As with all outpatientbased UK exercise training programmes, the majority of sessions were supervised by a rehabilitation physiotherapist. It was delivered as a bespoke and personalised intervention that was modified based on the response of the individual patient to exercise. The intervention consisted of two directly supervised exercise sessions and one independent unsupervised session per week over 6 weeks. Importantly, there was a standard operation procedure for delivery of the multimodal exercise therapies to ensure uniform delivery of the intervention. More specifically, the training schedule for the rehabilitation physiotherapists included targeted exercise treatments based on the physical capability of the patient with an instruction to deliver goaldirected therapy and progress the patient through the exercises over multiple sessions. The detail of the dose, duration and frequency of the exercise therapy were recorded to permit the investigators to interpret the relationship between these factors and outcome in terms of PF. This is

Division of Pulmonary, Critical Care and Sleep Medicine, University of Kentucky School of Medicine, Lexington, Kentucky, USA

Correspondence to Dr Peter E Morris, Division of Pulmonary, Critical Care and Sleep Medicine, University of Kentucky School of Medicine, 740 South Limestone, Lexington, KY 40536, USA; peter.morris@uky.edu the strict controlled approach that we would expect to be used in a drug study, but appears to be absent from many of the exercise therapy and rehabilitation studies.

As REVIVE 5 was focused on exercise therapy, and in line with previous studies of exercise therapy, the primary end point of REVIVE was the PF subscale of the Short Form-36 following 6 weeks of treatment. Disappointingly, the primary outcome did not demonstrate a difference between the intervention and control group but interestingly a number of the secondary outcomes showed a difference favouring the exercise therapy intervention. This deserves comment and indeed we should be cautious in our interpretation of these data. As a number of others in the field have highlighted, care should be taken when interpreting the data from clinical trials, in particular, when there are multiple secondary end point measurements. In general, ICU and post-ICU, exercise and rehabilitation studies have multiple subjective and objective measurements, which are subject to reporting bias. Investigators should be diligent in their approach and indeed they should not to limit their discussion to the positive secondary outcomes, as is common, but the investigators should detail the reasons that provide a rationale as to the failure to show a difference between the intervention and control groups in terms of the primary outcome. A common theme for clinical trialists currently is to state that there are 'no negative trials' and a tendency to categorise these 'no negative trials' as 'neutral trials'. Trials, whether 'negative' or 'neutral', still provide a prospectively gathered robust cohort dataset that always inform researchers in the field. Importantly, the investigators of the REVIVE feasibility study ${ }^{5}$ have been careful in their discussion of the primary outcome and they have given appropriate weight to the secondary outcomes. As optimists in an area that needs to find effective treatments for an increasing number of debilitated ICU survivors, the interpretation of $\operatorname{REVIVE}^{5}$ as a feasibility study provides a foundation to design and deliver a robust exercise intervention trial following critical illness.

The data from previous rehabilitation trials targeting post-ICU survivors have reported differing time points to start treatment from ICU admission, when the patient is transferred from the ICU to the ward, to several weeks after hospital discharge. $^{1-9}$ None of these trials, whether in the acute or postacute stage, has shown an improvement in outcome in terms of exercise capacity or PF. The REVIVE investigators ${ }^{5}$ delivered intervention in the outpatient setting, which allowed standardisation in the duration of the intervention delivered. Importantly, this design avoided the intervention being artificially altered based on the length of hospital stay, which is variable based on a number of clinical and non-clinical factors. Previous rehabilitation trials in this area have had been limited in their design as they only delivered the intervention as an inpatient, limiting the duration of intervention delivery.

An essential component of any clinical trial is to ensure that the intervention is delivered and adequately reported, as a failure to show a difference between the intervention and control group could be misinterpreted as a failure of the intervention rather than a failure of delivery of the intervention. The REVIVE investigators ${ }^{5}$ included an a priori definition of study adherence, which defined the number of sessions that needed to be completed by an individual to be considered as adherent to the protocol. This allows clinical trialists to consider intention-to-treat as well as per-protocol analysis and also to consider the relationship between the outcome variables and a 'dose effect', which provides greater detailed interpretation of the data. The investigators of the current study defined the minimum number of sessions necessary as $75 \%$ of therapy sessions offered. The investigators, as an a priori analysis plan, were able to report the effect of the intervention (per-protocol analysis) compared with the change in the intervention group as a whole (intention-to-treat analysis). The reasons for failure to deliver the interventional can then be discussed, which is an essential component of a well-designed feasibility study.

The target population of the REVIVE trial $^{5}$ was critically ill patients who had survived ICU after 4 days of mechanical ventilation. Importantly, the investigators defined their exclusion criteria, and enriched their target population, by excluding patients who would otherwise have been enrolled into an outpatient Pulmonary or Cardiac Rehabilitation Program. In the design of future trials, we need to be cognizant of the standard clinical management delivered as part of the ICU survivor's usual disease management plan. $^{9}$ In addition, we need to have 
threshold for inclusion such that patients who are on a trajectory of enhanced recovery should be excluded as these patients will reduce the effect size if they are randomised to the control group and enhance the effect size in randomised to treatment group. The same is true for those patients who are unlikely to achieve physical recovery. Either way, it will 'add noise' to the data. In addition, future work in this field needs to accommodate for the pre-ICU condition which can be managed at randomisation with minimising the groups based on specific chronic diseases such as chronic heart failure and COPD. This approach of target population enrichment will reduce the numbers of patients required to demonstrate a primary outcome signal.

If we can establish a clear approach to trial design for these post-ICU rehabilitation studies in terms of target population enrichment and intervention adherence and delivery, then we must also consider the standardisation of the outcomes measured. The ICU rehabilitation literature describes over 250 subjective and objective measurement tools reported in over 425 published studies. ${ }^{10} \mathrm{~A}$ priority is to characterise selection and definition of the outcomes used in existing trials of physical rehabilitation after critical illness and then obtain consensus on a core outcome set for future trials of post-ICU physical rehabilitation. ${ }^{11}$ This will allow studies to be compared across the recovery continuum from ICU admission, post-ICU discharge and posthospital discharge. The current outcome diversity negatively impacts on our ability to compare across studies and interpret the data which is contributing to the delay is translating the outcome from trials into routine clinical practice to improve the outcome of our patients.

The REVIVE trial ${ }^{5}$ has demonstrated it is feasible and safe to deliver exercise therapy to patients following critical illness in the outpatient setting. Furthermore, the investigators have shown that functional improvement can be achieved by delivering a specified dose and duration of an outpatient exercise programme in ICU survivors. Although this trial provides the framework for designing further clinical trials in this area, it is essential clinical researchers focus on the enriching the target population as well as developing a core outcome set to ensure that data from these trials can be compared and have immediate clinical impact. Supervised sessions with an operating procedure that standardises delivery of this complex intervention must be a priority. The REVIVE trial is another step in the right direction and we hope that the future rehabilitation trials will achieve improved function for post-ICU survivors.

Competing interests None declared.

Provenance and peer review Commissioned; externally peer reviewed.

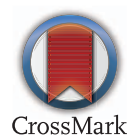

To cite Morris PE, Montgomery-Yates A. Thorax 2017;72:594-595.

Published Online First 7 March 2017

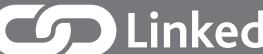

http://dx.doi.org/10.1136/thoraxjnl-2016-208723

Thorax 2017;72:594-595.

doi:10.1136/thoraxjnl-2016-209576

\section{REFERENCES}

1 Walsh TS, Salisbury LG, Merriweather JL, et al. Increased hospital-based physical rehabilitation and information provision after intensive care unit discharge: the RECOVER randomized clinical trial. JAMA Intern Med 2015;175:901-10.

2 Moss M, Nordon-Craft A, Malone D, et al. A randomized trial of an intensive physical therapy program for patients with acute respiratory failure. Am J Respir Crit Care Med 2016;193:1101-10.

3 Morris PE, Berry MJ, Files DC, et al. Standardized rehabilitation and hospital length of stay among patients with acute respiratory failure: a randomized clinical trial. JAMA 2016:315:2694-702.

4 Schaller SJ, Anstey M, Blobner M, et al. Early, goal-directed mobilisation in the surgical intensive care unit: a randomised controlled trial. Lancet 2016;388:1377-88.

5 McDowell K, O'Neill B, Blackwood B, et al. Effectiveness of an exercise programme on physical function in patients discharged from hospital following critical illness: a randomised controlled trial (the REVIVE trial). Thorax 2017;72:600-9.

6 Burtin C, Clerckx B, Robbeets C, et al. Early exercise in critically ill patients enhances short-term functional recovery. Crit Care Med 2009;37:2499-505.

7 Schweickert WD, Pohlman MC, Pohlman AS, et al. Early physical and occupational therapy in mechanically ventilated, critically ill patients: a randomised controlled trial. Lancet 2009;373:1874-82.

8 Denehy L, Skinner EH, Edbrooke L, et al. Exercise rehabilitation for patients with critical illness: a randomized controlled trial with 12 months of follow-up. Crit Care 2013;17:R156.

9 Greening NJ, Williams JE, Hussain SF, et al. An early rehabilitation intervention to enhance recovery during hospital admission for an exacerbation of chronic respiratory disease: randomised controlled trial. BMJ 2014;349:g4315.

10 Connolly B, Blackwood B, Hart N, et al. University of Liverpool. http://www.comet-initiative.org/studies/ details/288

11 Turnbull AE, Rabiee A, Davis WE, et al. Outcome measurement in ICU survivorship research from 1970 to 2013: a scoping review of 425 publications. Crit Care Med 2016;44:1267-77. 